\title{
Crianças pré-escolares e suas concepções de família ${ }^{1}$
}

\author{
Maria Auxiliadora Dessen² \\ Patrícia Cristina Campos Ramos \\ Universidade de Brasilia, Brasília-DF, Brasil
}

\begin{abstract}
Resumo: Para compreender o desenvolvimento e a diversidade da família contemporânea, faz-se necessário conhecer as concepções de família na perspectiva de crianças pré-escolares. Participaram deste estudo 33 crianças com idades de três, quatro e cinco anos: 15 do Grupo A (ambos os genitores com ocupação remunerada) e 18 do Grupo B (apenas o pai com ocupação remunerada). Os participantes foram entrevistados sobre suas concepções de família e referiram-se à composição familiar, à coabitação e ao cumprimento das funções de provedora, cuidadora e socializadora. As crianças consideraram como parte da composição da família: o núcleo familiar, membros da família extensa, padrinhos, amigos e animais de estimação. Porém, houve maior ênfase à função socializadora no Grupo B do que no Grupo A e as definições foram mais abstratas e complexas entre crianças de cinco anos. Os dados indicam que as concepções das crianças sobre os atuais modos de vida das famílias precisam ser investigadas em uma perspectiva longitudinal e transcultural.
\end{abstract}

Palavras-chave: pré-escolares, família, estrutura familiar.

\section{Preschool children and their family conceptions}

\begin{abstract}
To understand the development and diversity of the contemporary family, it is necessary to understand children's family conceptions. Thirty-three preschool children participated in the study (aged 3, 4, and 5 years old): 15 composed the Group A (both parents had a paid job), and 18 composed the Group B (only the father had a paid job). They were inquired about their conceptions of family and mentioned the following aspects: family composition, cohabitation, and the roles of provider, caregiver and socializing agent. Children included the nuclear family, extended family members, godparents, friends and pets in the composition of the family. However, the Group B emphasized the socializing function of the family more than the Group A. Moreover, children at aged 5 also provided more abstract and complex definitions than the other ones. The data indicates that children's conceptions on current family lifestyles need to be investigated in a longitudinal and cross-cultural perspective.
\end{abstract}

Keywords: preschoolers, family, family structure.

\section{Niños de edad preescolar y sus concepciones de la familia}

Resumen: Para comprender el desarrollo y la diversidad de la familia contemporánea, es necesario conocer las concepciones de los niños. En esta investigación participaron 33 niños de edad preescolar (3, 4 y 5 años), 15 en el Grupo A (ambos genitores tenían ocupación remunerada) y 18 en el grupo B (sólo el padre tenía ocupación remunerada). Los niños fueran entrevistados sobre sus concepciones de la familia, se refirieron a la composición familiar, la convivencia y el desempeño de las funciones de proveedor, cuidador y socializador. Los niños incluyeron en la composición de la familia tanto miembros de la familia nuclear, como de la familia amplia, padrinos, amigos y mascotas. Los resultados indican que en el grupo B se daba mayor énfasis a la función de socialización que en el Grupo A. También las concepciones fueron más abstractas y complejas en los niños de 5 años. Los datos indican la necesidad de investigar las concepciones de los niños sobre los actuales modos de vida de la familia desde una perspectiva longitudinal y trans-cultural.

Palabras clave: preescolares, familia, estructura familiar.

Pensar a família como parte de um contexto material, social e histórico conduz à compreensão de diferentes dinâmicas, papéis multidimensionais e complexos nela desempenhados, bem como das funções que lhe são atribuídas e que, de fato, são por ela exercidas. Assim, as concepções de

\footnotetext{
${ }^{1}$ Apoio: CNPq. Este artigo é derivado Dissertação de Mestrado defendida pela segunda autora, sob a orientação da primeira, no Programa de Pós-graduação em Processos de Desenvolvimento Humano e Saúde do Instituto de Psicologia da Universidade de Brasília.

${ }^{2}$ Endereço para correspondência

Maria Auxiliadora Dessen. Universidade de Brasília, Campus Universitário

'Darcy Ribeiro', Instituto de Psicologia - Departamento de Psicologia Escolar e do Desenvolvimento / PED, Laboratório de Desenvolvimento Familiar. CEP: 70.910-000, Brasília-DF, Brasil.E-mail: dessen@unb.br
}

família variam intra e entre culturas, em função de contextos sociais diversificados (Dessen \& Biasoli-Alves, 2001; Georgas, Berry, \& Kağitçibaşi, 2007).

A partir do século XVIII, a palavra família (famulus), criada para nomear a locação física de um grupo social de tribos latinas, passou a denominar uma unidade residencial e biológica comumente ligada ao agrupamento pai-mãecriança. Desde então, surgiram diferenciadas definições de família, em diversas áreas do conhecimento, com variações conceituais e teóricas (Cerqueira-Silva, Oliveira, \& Dessen, 2008; Valsiner, 2000). Além disso, mudanças estabelecidas nas sociedades contemporâneas ocidentais, sobretudo a partir da segunda metade do século XX, também influenciaram as concepções de família (Dessen \& Torres, 2002; Hodkin, Vacheresse, \& Buffett, 1996). 
A utilização de conceitos baseados apenas no modelo de família nuclear e nos laços de consangüinidade tornou-se inadequada, pois a versatilidade das famílias contemporâneas conduziu ao desenvolvimento de modelos conceituais mais abrangentes (Dessen \& Lewis, 1998; Stratton, 2003). Dentre tais modelos, destaca-se a definição intitulada 'ecopsicológica', em que a família é considerada "um grupo social especial, caracterizado pela intimidade e pelas relações intergeracionais" (Petzold, 1996, p. 39), sendo entendida, portanto, como um sistema complexo, composto por subsistemas integrados e interdependentes, e em inter-relação com o contexto sócio-histórico-cultural.

Se, por um lado, as definições científicas são importantes para nortear as pesquisas sobre família; por outro lado, as concepções de seus próprios membros tornam-se requisitos essenciais para a compreensão do funcionamento familiar. Tais concepções são oriundas das inter-relações estabelecidas na família, e desta com outros sistemas sociais, e influenciam significativamente o desenvolvimento do indivíduo (Hodkin e cols., 1996).

Assim, conhecer o que as pessoas pensam a respeito do que é ser "família" constitui o primeiro passo para a compreensão das relações familiares (Dessen \& Lewis, 1998). Porém, embora seja uma tendência nas pesquisas com famílias, atribuir importância às concepções de seus participantes, a criança raramente é incluída como fonte de informação (Hodkin e cols., 1996; Percy, 2003). Considerando ser ela um membro ativo do grupo familiar, é fundamental investigar as suas percepções e não apenas as percepções e interpretações dos adultos.

As crianças entendem e interpretam o que vêem não como os adultos o fazem. Elas desenvolvem seus conceitos, inclusive os referentes à família, baseadas em experiências de interação social, o que torna essencial a compreensão do seu ponto de vista (Montandon, 2005; Tudge, 2001). Apesar de serem poucos os estudos, especialmente brasileiros, sobre as concepções que as crianças têm de família, as contribuições científicas existentes são diversificadas e relevantes. Por exemplo, Moore, Bickhard e Cooper (1977) entrevistaram 84 crianças, cujos genitores viviam juntos ou eram divorciados, a respeito do entendimento de família. Os autores verificaram que crianças de famílias divorciadas mencionaram mais fatores emocionais e atividades para adultos, e utilizaram a composição para a formação da família com menor freqüência do que crianças cujos genitores viviam juntos. Elas consideraram família, principalmente, grupos compostos por dois genitores e uma criança, um par idoso e, em menor proporção, um casal novo sem crianças. Para aproximadamente metade delas, a maioria proveniente de famílias com genitores vivendo juntos, um único genitor e uma criança não formavam uma família.

Outro estudo foi o realizado por Powell, Wiltcher, Wedemeyer e Claypool (1981), que entrevistaram, individualmente, 84 crianças pré-escolares de três a seis anos de idade, que também pertenciam a famílias com genitores vivendo juntos ou divorciados. De suas respostas, foram destacadas as seguintes dimensões da família: composição, aspectos biológicos, funções domésticas e orientações. Como parte dos resultados, para crianças de ambos os tipos de família, a composição familiar mais citada continha, ao menos, dois genitores e uma criança, corroborando os resultados encontrados por Moore e cols. (1977).

Também normas e crenças parentais, idéias infantis e uso da linguagem no conceito de família, sob a perspectiva de crianças de oito a 14 anos, foram estudados por Morrow (1998). Nesse estudo era solicitado que a criança desenhasse e escrevesse sobre "o que é família" e completasse sentenças simples a respeito de tipos de família. As crianças definiram família em termos de papéis, relacionamentos e estrutura, não se restringindo apenas às relações biológicas ou ao núcleo familiar. Em suas definições de família, crianças com menos idade utilizaram termos mais concretos e citaram a existência de filhos, o casamento e a presença física no domicílio, além de enfatizarem a provisão e os cuidados por parte dos genitores. Aquelas de mais idade utilizaram uma linguagem mais generalizada e noções abstratas complexas que ultrapassavam as próprias experiências, e a qualidade dos relacionamentos mostrouse central para elas. Em geral, as crianças apresentaram uma visão pragmática da vida familiar e demonstraram conhecer a variedade de suas práticas e estruturas, com pouca discrepância entre a descrição da própria família. Elas apontaram como importantes: genitores, outros parentes e relacionamentos especiais, como amigos, padrinhos e animais de estimação.

Independentemente da fonte de informação, se adultos ou crianças, os resultados de pesquisas sobre a composição familiar dependem, em grande parte, da técnica empregada na coleta de dados (Dessen \& Lewis, 1998). Algumas técnicas são consideradas mais adequadas às explorações da opinião de crianças. Recentemente, pesquisadores têm associado narrativas às histórias/dilemas com bonecos, na tentativa de que as crianças, no final da história, resolvam o conflito proposto. Com essa técnica, Laible, Carlo, Torquati e Ontai (2004) investigaram as percepções de 74 crianças sobre os relacionamentos familiares e sugeriram que o clima emocional exerce impacto na construção das representações que, por sua vez, se refletem na conduta e nas interações sociais das crianças.

Além das narrativas, também os desenhos estão entre os instrumentos considerados apropriados para essas explorações. Por meio desta técnica, Roe, Bridges, Dunn e O’Connor (2006) investigaram representações de família feitas por 119 crianças com idade média de sete anos. Ao longo de dois anos, foram observadas estabilidades e mudanças quanto à inclusão/exclusão de membros na família 
e quanto às formas como eram agrupados nos desenhos. Os dados demonstraram consistência tanto nas representações quanto nas predições do ajustamento da criança. Crianças de menos idade tendiam a apresentar habilidades verbais e cognitivas relativamente imaturas, de forma que os autores concluiram que as percepções de crianças pequenas, a respeito da vida familiar e de suas transições, necessitavam ser melhor investigadas.

Shermerhorn, Cummings e Davies (2008) examinaram, em 132 crianças do jardim da infância, processos de influência da família, por meio de tarefas de histórias-núcleo para completar. Os resultados deste estudo longitudinal, apontado como sendo pioneiro no uso de respostas representacionais dadas por crianças a respeito da interligação da relação conjugal com as relações de apego, possibilitaram verificar que as representações das crianças eram interdependentes e inseridas na complexa dinâmica dos relacionamentos e de suas múltiplas influências.

Apesar da importância e do alcance dos estudos apresentados, o processo de desenvolvimento do conceito de família para as crianças ainda é pouco conhecido (Percy, 2003). Portanto, este estudo teve como objetivo compreender o que as crianças pré-escolares pensam a respeito do que é família e do que faz a família no seu dia-a-dia, destacando as similaridades e/ou diferenças: a) quando ambos os genitores ou apenas o pai exerce $(m)$ ocupação remunerada e b) entre crianças de três, quatro e cinco anos de idade.

\section{Método}

\section{Participantes}

Este estudo contou com a participação de 33 crianças pré-escolares e suas famílias, residentes em diversas regiões administrativas do Distrito Federal. Pai e mãe biológicos eram casados ou coabitavam desde o nascimento da criança participante, independentemente de ser a primeira união do casal, e tinham até três filhos. As crianças foram distribuídas em dois grupos: Grupo A ( $\mathrm{n}=15)$, com ambos os genitores exercendo ocupação remunerada, e Grupo B $(n=18)$, com apenas o pai exercendo tal ocupação. Havia 10 crianças de três anos $(A: n=8 ; B: n=2) ; 16$ de quatro anos $(A: n=2 ; B$ : $n=14)$ e sete de cinco anos $(A: n=5 ; B: n=2)$. Quanto ao gênero, 15 eram meninas $(A: n=8 ; B: n=7)$ e 18 meninos $(A$ : $\mathrm{n}=8 ; \mathrm{B}: \mathrm{n}=7$ ).

As mães tinham entre 18 e 41 anos e os pais, entre 21 e 55 anos, com frequência maior de genitores entre 21 e 30 anos no Grupo B e entre 31 e 40 anos no Grupo A. Evidenciou-se maior escolaridade das mães. Mães e pais com ocupações remuneradas exerciam atividades diversificadas e possuíam rendimentos variados (de $1 / 2$ a 15 salários mínimos), com média de 4,7 salários mínimos por família e maior contribuição financeira por parte dos pais.

\section{Instrumentos}

Questionário Sociodemográfico da Família (Dessen, 1999). O questionário foi respondido pelas mães. Por meio deste foram obtidos dados relativos à caracterização das famílias participantes, como: idade e escolaridade dos familiares, estado civil e ocupação dos genitores, divisão de tarefas domésticas, estrutura de suporte social e atividades de rotina.

Entrevista semi-estruturada com a criança. Por meio da entrevista foram investigadas as concepções das crianças sobre a família. Na primeira parte, utilizou-se um cenário contendo uma espaçonave de brinquedo e dois bonecos, representando extraterrestres que, supostamente, não sabiam o que era uma família, o que lhes seria explicado pela criança. $\mathrm{O}$ procedimento teve como objetivo tornar a entrevista lúdica e atrativa. À criança era solicitado que respondesse, em momentos distintos: a) o que era, e b) o que fazia - uma família, uma boa família e uma família má.

$\mathrm{Na}$ segunda parte da entrevista foram apresentadas às crianças duas pequenas histórias, que continham aspectos característicos e definidores do conceito de família, a fim de verificar sua opinião. A primeira história descrevia um grupo composto por homens e mulheres que viviam na mesma casa, faziam refeições, saiam juntos e trocavam presentes, uma das mulheres cozinhava para todos e um dos homens fazia compras, eles se amavam, mas não estavam totalmente envolvidos. Na segunda história, o grupo era composto por um homem e três mulheres que moravam na mesma casa, se gostavam, faziam uma refeição juntos na semana, porém não passavam muito tempo juntos, uma das mulheres cuidava da casa e o homem fazia compra para todos. Quando necessário, o entrevistador fazia perguntas com intuito de esclarecer ou de que a criança justificasse sua resposta, complementando-a. A história era repetida, caso a criança demonstrasse incompreensão.

\section{Considerações éticas}

A coleta de dados era iniciada pelo preenchimento, com os genitores, do Termo de Consentimento Livre e Esclarecido.

\section{Procedimentos \\ Coleta de dados}

As famílias das crianças foram contatadas por intermédio de escolas públicas de educação infantil. Devido ao baixo número de famílias dentro do perfil para a pesquisa, elas também foram localizadas por indicações dos próprios participantes. Por contato telefônico, era confirmada a disponibilidade em participar, apresentados os objetivos do estudo e combinada uma visita à casa da família, com duração média de quatro horas. A entrevista semi-estruturada com cada criança durou cerca de 20 a 40 minutos e ocorreu em uma única sessão. 


\section{Análise dos dados}

As respostas do Questionário Sociodemográfico da Família foram submetidas à análise estatística descritiva, sendo calculadas as frequências absolutas e os percentuais.

As verbalizações das crianças foram submetidas a três etapas de uma análise:

Etapa I - Preparação do material. Gravadas em áudio, as entrevistas foram identificadas e digitalizadas, para aperfeiçoamento da escuta. A transcrição foi realizada na íntegra.

Etapa II - Análise de conteúdo das entrevistas. As verbalizações das crianças foram submetidas à técnica de Análise de Conteúdo (AC). Frente às especificidades da entrevista realizada com crianças, houve necessidade de algumas adaptações no modelo proposto por Dessen e Cerqueira-Silva (2009), para o desenvolvimento de sistemas de categorias, de forma que os seguintes passos foram seguidos:

(1) Pré-análise: seleção e exploração do material, iniciadas por uma leitura exaustiva de cada entrevista. Mostrouse necessário um reagrupamento das verbalizações dentro da proposta de cada questão, visto que, muitas vezes, as crianças se remetiam de uma questão à outra, ou de um aspecto a outro de uma mesma questão.

(2) Codificação: exploração do material, com a identificação dos temas (unidade de análise) referentes às verbalizações das crianças.

(3) Identificação, agrupamento e categorização de temas semelhantes, ou relacionados entre si. Cada agrupamento resultou em uma categoria, obedecendo aos critérios de exclusão mútua, homogeneidade e pertinência.

Etapa III - Elaboração da versão final das categorias. O processo de categorização pressupõe a elaboração de várias versões do sistema (Dessen \& Cerqueira-Silva, 2009). Sendo assim, três versões com várias modificações foram re-elaboradas no presente estudo, até que uma versão final da categorização fosse obtida, por meio dos seguintes passos:

(1) Definição e validação das categorias empíricas. Devido à complexidade do tema e diversidade nas respostas, os processos de categorização e definição foram construídos e revisados com a colaboração dos pesquisadores do Laboratório de Desenvolvimento Familiar, em busca de maior validade e objetividade do sistema definitivo de categorias.

(2) Definição e tabela de categorias. Devido à diversidade das respostas, foram elaboradas tabelas que incluíam as categorias, os temas e todas as verbalizações das crianças, assim como suas freqüências. Uma classificação foi atribuída a cada categoria, a partir de um conjunto de relatos que expressavam e explicavam o que nela estava contido. $\mathrm{O}$ sistema de categorias definitivo levou em consideração cada dimensão abordada no roteiro de entrevista: a) o que é, e b) o que faz - uma família, uma boa família e uma família má.

(3) Tabulação dos dados. As categorias criadas com base nas verbalizações das crianças foram também examinadas em termos de freqüências absolutas e relativas, visando a sua quantificação e comparação entre os grupos.

\section{Resultados}

Nesta seção, serão descritas as concepções das crianças, por grupo (A e B) e por idade (três, quatro e cinco anos), a respeito do que é família e do que ela faz, também quando considerada como sendo boa e má. As freqüências apresentadas referem-se ao total de verbalizações dos participantes por grupo (A: $n=15 ; B: n=18)$ ou por idade $(3: n=104: n=16$; $5: \mathrm{n}=7)$. Em seguida, serão sintetizadas as opiniões das crianças frente à apresentação de aspectos característicos e definidores do conceito de família.

\section{As crianças e suas concepções de família}

O que é família

Em suas verbalizações, as crianças citaram dois tipos de família: as do mundo todo - "Tem um montão de família assim que tem no mundo todo"; e as do céu, de Deus - "Tem um monte de família que é Deus". Por vezes, a família foi apenas identificada, nomeada elou caracterizada - "Uma família do meu pai" / "Família é uma coisa legal". Em relação à composição familiar, foi utilizado o critério de quantidade, sendo a família formada por: a) apenas "uma pessoa"; b) "duas"; ou c) várias pessoas - "É ter uma família bem grande".

A composição familiar também foi descrita pelas crianças de acordo com suas relações a) biológicas - "É filho, pai, mãe e filha" / "É a nossa mãe, nosso pai, nossa tia. É nosso tio, nossa avó, nosso avô"; b) biológicas e não biológicas, com padrinhos, amigos, pessoas pobres e/ou animais de estimação; e c) biológicas e outras, em que não foi possível identificar a ligação com a criança - "É... a minha mãe, o meu pai. (...) Minha tia. E aquele Bebecuri”.

Foram descritas duas formas de coabitação: a) cada família habitando um domicílio - "[Um monte de pessoas] que mora em cada casa"; e b) familiares em diferentes domicílios/localidades - "Tem um punhado de pessoas da minha família que moram aqui em Brasília, tem um que mora em Piataba e o resto no Pará". Em relação aos sentimentos, foram encontrados: os de pertencimento (ou não) à família, pois a criança afirmou ou negou tê-la; de gostar e de ficar feliz. Quanto à função socializadora, a família educa os filhos e promove o lazer e/ou o contato social - "Recebe os amigos, recebe os primos, recebe toda a família".

As crianças de ambos os grupos (A: $f=28 \%$; B: $f=$ $23,6 \%)$ verbalizaram a respeito das relações biológicas na composição da família. Também houve considerável percentual de definições que incluíam relações não biológicas (A: $f=16 \%$; B: $f=14,7 \%$ ). Elas destacaram aspectos da função socializadora da família, especialmente no Grupo B $(14,7 \%)$. Estes dados são visualizados na Tabela 1. 
Tabela 1

Categorização das verbalizações sobre o que é família

\begin{tabular}{|c|c|c|c|c|c|c|}
\hline \multirow{3}{*}{ Categorias/temas/subtemas } & \multicolumn{4}{|c|}{ Grupos } & & \\
\hline & \multicolumn{2}{|c|}{$\begin{array}{c}\mathbf{A} \\
(\mathrm{n}=15)\end{array}$} & \multicolumn{2}{|c|}{$\begin{array}{c}\text { B } \\
(\mathrm{n}=18)\end{array}$} & \multicolumn{2}{|c|}{$\begin{array}{c}\text { Total } \\
(\mathbf{N}=33)\end{array}$} \\
\hline & $f$ & $\%$ & $f$ & $\%$ & $f$ & $\%$ \\
\hline \multicolumn{7}{|l|}{ Identificação da família } \\
\hline \multicolumn{7}{|l|}{ tipos } \\
\hline do mundo todo & - & - & 1 & 2,9 & 1 & 1,7 \\
\hline do céu, de Deus & - & - & 1 & 2,9 & 1 & 1,7 \\
\hline localiza/nomeia/caracteriza & 2 & 8,0 & 2 & 5,9 & 4 & 6,8 \\
\hline subtotal & 2 & 8,0 & 4 & 11,8 & 6 & 10,2 \\
\hline \multicolumn{7}{|l|}{ Composição familiar } \\
\hline \multicolumn{7}{|l|}{ quantidade de pessoas } \\
\hline uma & 1 & 4,0 & 2 & 5,9 & 3 & 5,1 \\
\hline duas & & & 1 & 2,9 & 1 & 1,7 \\
\hline várias/família grande & 2 & 8,0 & 3 & 8,8 & 5 & 8,5 \\
\hline \multicolumn{7}{|l|}{ quem faz parte } \\
\hline relações biológicas & 7 & 28,0 & 8 & 23,6 & 15 & 25,3 \\
\hline relações biológicas e não biológicas & 4 & 16,0 & 5 & 14,7 & 9 & 15,2 \\
\hline relações biológicas e não identificadas & & & 1 & 2,9 & 1 & 1,7 \\
\hline subtotal & 14 & 56,0 & 20 & 58,8 & 34 & 57,5 \\
\hline \multicolumn{7}{|l|}{ Coabitação } \\
\hline cada família habita um domicílio & 2 & 8,0 & - & - & 2 & 3,4 \\
\hline familiares em diferentes domicílios & 1 & 4,0 & - & - & 1 & 1,7 \\
\hline subtotal & 3 & 12,0 & - & - & 3 & 5,1 \\
\hline \multicolumn{7}{|l|}{ Sentimentos } \\
\hline \multicolumn{7}{|l|}{ pertencimento à família } \\
\hline afirma ter & 1 & 4,0 & 1 & 2,9 & 2 & 3,4 \\
\hline nega ter & 1 & 4,0 & & & 1 & 1,7 \\
\hline ficar feliz/gostar & 1 & 4,0 & 1 & 2,9 & 2 & 3,4 \\
\hline subtotal & 3 & 12,0 & 2 & 5,9 & 5 & 8,5 \\
\hline \multicolumn{7}{|l|}{ Aquela que cumpre a função } \\
\hline \multicolumn{7}{|l|}{ socializadora } \\
\hline educa & & & 3 & 8,8 & 3 & 5,1 \\
\hline promove lazer/contato social & 1 & 4,0 & 2 & 5,9 & 3 & 5,1 \\
\hline subtotal & 1 & 4,0 & 5 & 14,7 & 6 & 10,2 \\
\hline \multicolumn{7}{|l|}{ Não definida } \\
\hline dá respostas vagas & 1 & 4,0 & 1 & 2,9 & 2 & 3,4 \\
\hline diz não saber & 1 & 4,0 & 2 & 5,9 & 3 & 5,1 \\
\hline subtotal & 2 & 8,0 & 3 & 8,8 & 5 & 8,5 \\
\hline Total & 25 & 100 & 34 & 100 & 59 & 100 \\
\hline
\end{tabular}

As crianças de três anos foram as que menos responderam sobre o que é família e, quando o fizeram, destacaram sua composição, enfatizando, particularmente, as relações biológicas. Por outro lado, todas as crianças de cinco anos conceituaram a família. A inclusão de relações não-biológicas ocorreu, progressivamente, entre crianças de quatro e cinco anos.
O que uma familia faz?

Quanto ao que a família faz, foram diversificadas as respostas. A família categorizada como provedora é aquela que dá e/ou compra coisas - "Compra brinquedos"; a cuidadora "fica com os filhos". A família socializadora educa, favorecendo a autonomia - "Deixa os filho ir 'pa' escola sozinho"; ensina atividades domésticas; promove brincadeiras e 
atividades de lazer - "Faz a gente brincar" / "Leva pra passear”. Para as crianças, a família é afetiva sendo carinhosa com elas. Foram destacados, também, alguns hábitos da família, como não fazer maldade e orar.

Em ambos os grupos, as crianças enfatizaram o cumprimento da função de socializadora (A: $f=40 \%$; B: $f=56,2 \%$ ) e da função de provedora (A: $f=20 \%$; B: $f=18,8 \%$ ). Hábitos da família foram citados no Grupo A (20\%) e houve famílias não definidas, em ambos os grupos (quando a criança respondia que não sabia ou não lembrava). A categorização das verbalizações a respeito do que uma família faz é ilustrada na Tabela 2 .

Tabela 2

Categorização das verbalizações sobre o que uma família faz

\begin{tabular}{|c|c|c|c|c|c|c|}
\hline \multirow{3}{*}{ Categorias/temas/subtemas } & \multicolumn{4}{|c|}{ Grupos } & & \\
\hline & \multicolumn{2}{|c|}{$\begin{array}{c}A \\
(n=15)\end{array}$} & \multicolumn{2}{|c|}{$\begin{array}{c}B \\
(n=18)\end{array}$} & \multicolumn{2}{|c|}{$\begin{array}{c}\text { Total } \\
(\mathbf{N}=33)\end{array}$} \\
\hline & $f$ & $\%$ & $f$ & $\%$ & $f$ & $\%$ \\
\hline \multicolumn{7}{|l|}{ Cumpre sua função } \\
\hline \multicolumn{7}{|l|}{ provedora } \\
\hline dá/compra coisas & 2 & 20,0 & 3 & 18,8 & 5 & 19,3 \\
\hline \multicolumn{7}{|l|}{ cuidadora } \\
\hline cuida da criança & - & - & 1 & 6,2 & 1 & 3,8 \\
\hline \multicolumn{7}{|l|}{ socializadora } \\
\hline educa & - & - & 1 & 6,2 & 1 & 3,8 \\
\hline ensina atividades domésticas & - & - & 1 & 6,2 & 1 & 3,8 \\
\hline promove brincadeiras & 1 & 10,0 & 3 & 18,8 & 4 & 15,4 \\
\hline promove lazer & 3 & 30,0 & 4 & 25,0 & 7 & 26,9 \\
\hline subtotal & 4 & 40,0 & 9 & 56,2 & 13 & 49,9 \\
\hline \multirow[t]{2}{*}{ A criança descreve } & & & & & & \\
\hline & 2 & 20,0 & - & - & 2 & 7,7 \\
\hline \multicolumn{7}{|l|}{ Não definido } \\
\hline dá respostas vagas & 2 & 20,0 & - & - & 2 & 7,7 \\
\hline diz não saber & & & 3 & 18,8 & 3 & 11,6 \\
\hline subtotal & 2 & 20,0 & 3 & 18,8 & 5 & 19,3 \\
\hline Total & 10 & 100 & 16 & 100 & 26 & 100 \\
\hline
\end{tabular}

Ao verbalizarem sobre o que uma família faz, as crianças destacaram algumas funções da família: aos quatro anos $(41,7 \%)$ e aos cinco anos $(58,3 \%)$, a função de socializadora, e das duas crianças de três anos que responderam à questão, uma destacou a função de socializadora e outra, a de provedora.

\section{O que é uma família boa? E uma família má?}

Para as crianças, a própria família (ou um de seus integrantes) foi identificada como sendo boa - "É o meu pai (...). Minha mãe.... Minha avó". Elas consideram como boa família aquela que dá e/ou compra coisas (provedora) "Que compra bicicleta"; protege e alimenta (cuidadora) - "É quando "tá' protegendo os filhos" / "Quando 'tá' dando comida pros filhos"; recebe as pessoas (socializadora) - "Recebe todo mundo, que deixa os amigos entrarem"; e que gosta de todos (afetiva). Também foram citados alguns hábitos da boa família, como não matar bichos.
Quanto à família má, as crianças a identificaram, por vezes em um de seus membros - "É o pai"; ou apenas repetiram "má" ou sinônimos - "Uma pessoa que é muito "mau". Para estas crianças, uma família má é aquela que não cumpre adequadamente suas funções de a) provedora - "Uma família ruim é aquela que não dá nada de bom pra criança"; b) cuidadora - "Não deixa as 'pessoa' dormir"; c) socializadora, pois bate e morde - "Uma pessoa que (...) bate na gente, uma pessoa que morde"; não recebe as pessoas - "Ela (...) não recebe as crianças com carinho, nem amigos"; e não brinca e/ou não promove atividades de lazer - "Não faz eu brincar" / "Uma família que não leva pra passear". Foi ainda citada como má a família que tem maus hábitos.

Em relação aos grupos, houve uma maior freqüência de verbalizações no Grupo A, em que boa família seria aquela que cumpre a função de cuidadora, ao proteger e alimentar $(33,3 \%)$, enquanto, no Grupo B, as crianças verbalizaram mais sobre sua identificação $(60 \%)$. 
Em se tratando das frequências de verbalizações sobre a família má, houve pouca diferenciação entre grupos. Ela é aquela que não cumpre adequadamente as funções de provedora (A $f=16,7 \%$ ), cuidadora (B $f=20 \%$ ) e socializadora (A $f=50 \%$; B $f=20 \%$ ). Em algumas verbalizações, as crianças citaram maus hábitos da família má ( $\mathrm{A} f=16,7 \%$; B $f=20 \%$ ), havendo também quem apenas a identificou (A $f=16,7 \%$; B $f=20 \%$ ). A Tabela 3 corresponde à categorização das verbalizações das crianças, por grupo, às questões: "o que é uma boa família" e "o que é uma família má".

Tabela 3

Categorização das verbalizações sobre o que é uma boa família e uma família má

\begin{tabular}{|c|c|c|c|c|c|c|}
\hline \multirow{3}{*}{ Categorias } & \multicolumn{4}{|c|}{ Grupos } & & \\
\hline & \multicolumn{2}{|c|}{$\begin{array}{c}\mathbf{A} \\
(n=15)\end{array}$} & \multicolumn{2}{|c|}{$\begin{array}{c}B \\
(n=18)\end{array}$} & \multicolumn{2}{|c|}{$\begin{array}{c}\text { Total } \\
(\mathbf{N}=33)\end{array}$} \\
\hline & $f$ & $\%$ & $f$ & $\%$ & $f$ & $\%$ \\
\hline \multicolumn{7}{|l|}{ Família boa } \\
\hline \multicolumn{7}{|l|}{ Identificação } \\
\hline é a própria família & & & 1 & 20,0 & 1 & 9,1 \\
\hline são integrantes da família & 1 & 16,6 & 1 & 20,0 & 2 & 18,2 \\
\hline apenas repete 'boa' ou sinônimos & & & 1 & 20,0 & 1 & 9,1 \\
\hline subtotal & 1 & 16,6 & 3 & 60,0 & 4 & 36,4 \\
\hline \multicolumn{7}{|l|}{ É aquela que cumpre a função } \\
\hline \multicolumn{7}{|l|}{ provedora } \\
\hline dá/compra coisas & 1 & 16,7 & 1 & 20,0 & 2 & 18,2 \\
\hline \multicolumn{7}{|l|}{ cuidadora } \\
\hline protege/alimenta & 2 & 33,3 & - & - & 2 & 18,2 \\
\hline \multicolumn{7}{|l|}{ socializadora } \\
\hline recebe as pessoas & 1 & 16,7 & - & - & 1 & 9,1 \\
\hline \multicolumn{7}{|l|}{ afetiva } \\
\hline gosta de todos & 1 & 16,7 & - & - & 1 & 9,1 \\
\hline subtotal & 5 & 83,4 & 1 & 20,0 & 6 & 54,6 \\
\hline \multicolumn{7}{|l|}{ É aquela que tem hábitos } \\
\hline altruístas/ecológicos (não mata bichos) & & & 1 & 20,0 & 1 & 9,0 \\
\hline Subtotal família boa & 6 & 100 & 5 & 100 & 11 & 100 \\
\hline \multicolumn{7}{|l|}{ Família má } \\
\hline \multicolumn{7}{|l|}{ Identificação } \\
\hline é um integrante da família & 1 & 16,7 & - & - & 1 & 9,1 \\
\hline apenas repete 'má' ou sinônimos & & & 1 & 20,0 & 1 & 9,1 \\
\hline subtotal & 1 & 16,7 & 1 & 20,0 & 2 & 18,2 \\
\hline \multicolumn{7}{|c|}{$\begin{array}{l}\text { É aquela que não cumpre adequadamente a função } \\
\text { provedora }\end{array}$} \\
\hline não dá nada de bom & 1 & 16,7 & - & - & 1 & 9,1 \\
\hline \multicolumn{7}{|l|}{ cuidadora } \\
\hline não deixa dormir & - & - & 1 & 20,0 & 1 & 9,1 \\
\hline \multicolumn{7}{|l|}{ socializadora } \\
\hline pune (morde/bate) & - & - & 1 & 20,0 & 1 & 9,1 \\
\hline não promove brincadeiras & 1 & 16,7 & & & 1 & 9,1 \\
\hline não promove lazer & & & 1 & 20,0 & 1 & 9,1 \\
\hline não recebe as pessoas & 2 & 33,3 & & & 2 & 18,2 \\
\hline subtotal & 4 & 66,6 & 3 & 60,0 & 7 & 63,6 \\
\hline \multicolumn{7}{|l|}{ É aquela que tem maus hábitos } \\
\hline é ladrão & - & - & 1 & 20,0 & 1 & 9,1 \\
\hline há conflitos/desacordos & 1 & 16,7 & & & 1 & 9,1 \\
\hline subtotal & 1 & 16,7 & 1 & 20,0 & 2 & 18,2 \\
\hline Subtotal família má & 6 & 100 & 5 & 100 & 11 & 100 \\
\hline
\end{tabular}


Quanto às idades, a única criança de três anos que respondeu a respeito da boa família apenas a identificou; crianças de quatro anos citaram a boa família como aquela que cumpre, principalmente, a função de cuidadora $(28,6 \%)$; aos cinco anos foram destacadas, em igual proporção $(33,3 \%)$, as funções de provedora, socializadora e afetiva. Hábitos da boa família foram citados por crianças de quatro anos (14,2\%).

Em relação à família má, uma criança de três anos identificou-a em um integrante da família e outra enfatizou o não cumprimento adequado da função de socializadora. Crianças de quatro anos também verbalizaram a respeito do não cumprimento adequado dessa função (40\%), em diversas atividades. Poucas crianças de cinco anos responderam à questão, predominando, em suas definições, a família má como aquela que não cumpre adequadamente a função de socializadora $(50 \%)$.

\section{O que faz uma boa família? E uma família má?}

Para as crianças, o que uma boa família faz está relacionado ao cumprimento das funções de: a) provedora, dando e/ou comprando coisas para o filho; b) cuidadora, alimentando e cuidando de tudo - "Cuida de tudo na família"; c) socializadora, ao educar (favorecendo a autonomia, não castigando, não batendo) - “Deixa os 'filho é fica' lá fora de biciqueta" / "Não deixa o filho de castigo" / "Não bate"; e brincando e/ou promovendo atividades de lazer - "Leva 'pra passiá' lá pro parquinho" e d) afetiva, gostando e/ou amando a criança - "Gosta do filho". Por vezes, a boa família foi apenas identificada pela criança, que repetiu a expressão "boa" ou um sinônimo.

Em relação ao que faz uma família má, na opinião das crianças, ela não cumpre adequadamente as funções de: a) provedora - "Que não dá nada pras crianças"; b) socializado$r a$, pois pune - "Porque quando os filhos pega as coisas dela, sem pedir, aí ela morde" / "E também bate nas crianças"; e c) afetiva - "É [ruim] pros filhos". Uma das crianças destacou que a própria família não fazia o que faz uma família má; outras salientaram maus hábitos da família. A maior proporção de verbalizações foi sobre o não cumprimento adequado da função de socializadora.

A respeito do que faz uma boa família, as crianças do Grupo A destacaram o cumprimento adequado das funções de provedora $(27,3 \%)$ e de socializadora $(27,2 \%)$ e as do Grupo B, o cumprimento da função de socializadora (45\%). Quanto ao que faz uma família má, crianças de ambos os grupos destacaram que ela não cumpre adequadamente a função de socializadora (A: $f=61,5 \%$; B: $f=36,3)$, especialmente por punir. A categorização das verbalizações das crianças sobre o que faz uma família boa e uma família má, por grupo, encontra-se descrita na Tabela 4.

Tabela 4

Categorização das verbalizações sobre o que faz uma família boa e o que faz uma família má

\begin{tabular}{|c|c|c|c|c|c|c|}
\hline \multirow{3}{*}{ Categorias, temas e subtemas } & \multicolumn{4}{|c|}{ Grupos } & \multirow{2}{*}{\multicolumn{2}{|c|}{$\begin{array}{c}\text { Total } \\
(\mathbf{N}=\mathbf{3 3})\end{array}$}} \\
\hline & \multicolumn{2}{|c|}{$\begin{array}{c}\mathbf{A} \\
(\mathrm{n}=15)\end{array}$} & \multicolumn{2}{|c|}{$\begin{array}{c}\text { B } \\
(n=18)\end{array}$} & & \\
\hline & $f$ & $\%$ & $f$ & $\%$ & $f$ & $\%$ \\
\hline \multicolumn{7}{|l|}{ Família boa } \\
\hline \multicolumn{7}{|l|}{ Identificação } \\
\hline apenas repete 'boa' ou sinônimos & 1 & 9,1 & 1 & 9,1 & 2 & 9,1 \\
\hline \multicolumn{7}{|l|}{ Cumpre sua função } \\
\hline \multicolumn{7}{|l|}{ Provedora } \\
\hline dá/compra coisas & 3 & 27,3 & - & - & 3 & 13,6 \\
\hline \multicolumn{7}{|l|}{ Cuidadora } \\
\hline alimenta & 2 & 18,2 & - & - & 2 & 9,1 \\
\hline cuida de tudo & - & - & 1 & 9,1 & 1 & 4,5 \\
\hline \multicolumn{7}{|l|}{ Socializadora } \\
\hline educa & 2 & 18,2 & 2 & 18,2 & 4 & 18,2 \\
\hline promove lazer & 1 & 9,0 & 3 & 27,3 & 4 & 18,2 \\
\hline \multicolumn{7}{|l|}{ Afetiva } \\
\hline gosta, ama a criança & 2 & 18,2 & 2 & 18,2 & 4 & 18,2 \\
\hline \multicolumn{7}{|l|}{ Não definido } \\
\hline dá respostas vagas & - & - & 1 & 9,1 & 1 & 4,5 \\
\hline diz não saber & - & - & 1 & 9,1 & 1 & 4,5 \\
\hline subtotal & - & - & 2 & 18,2 & 2 & 9,1 \\
\hline Subtotal família boa & 11 & 100 & 11 & 100 & 22 & 100 \\
\hline
\end{tabular}


Tabela 4

\begin{tabular}{|c|c|c|c|c|c|c|}
\hline \multirow{3}{*}{ Categorias, temas e subtemas } & \multicolumn{4}{|c|}{ Grupos } & \multirow{2}{*}{\multicolumn{2}{|c|}{$\begin{array}{c}\text { Total } \\
(\mathbf{N}=\mathbf{3 3})\end{array}$}} \\
\hline & \multicolumn{2}{|c|}{$\begin{array}{c}A \\
(n=15)\end{array}$} & \multicolumn{2}{|c|}{$\begin{array}{c}\text { B } \\
(n=18)\end{array}$} & & \\
\hline & $f$ & $\%$ & $f$ & $\%$ & $f$ & $\%$ \\
\hline \multicolumn{7}{|l|}{ Família má } \\
\hline \multicolumn{7}{|l|}{ Identificação da família (que não é) má } \\
\hline a própria família não é má & - & - & 1 & 9,1 & 1 & 4,1 \\
\hline \multicolumn{7}{|l|}{ Não cumpre adequadamente sua função } \\
\hline \multicolumn{7}{|l|}{ Provedora } \\
\hline não dá/não compra coisas & 2 & 15,4 & 1 & 9,1 & 3 & 12,5 \\
\hline \multicolumn{7}{|l|}{ Socializadora } \\
\hline mata & 1 & 7,7 & & & 1 & 4,2 \\
\hline pune & 7 & 53,8 & 3 & 27,2 & 10 & 41,6 \\
\hline não passeia & & & 1 & 9,1 & 1 & 4,2 \\
\hline \multicolumn{7}{|l|}{ Afetiva } \\
\hline é ruim para os filhos & 1 & 7,7 & - & - & 1 & 4,2 \\
\hline subtotal & 11 & 84,6 & 5 & 45,4 & 16 & 66,7 \\
\hline \multicolumn{7}{|l|}{ A criança descreve hábitos da família má } \\
\hline bagunça/quebra coisas & 1 & 7,7 & 1 & 9,1 & 2 & 8,3 \\
\hline não altruístas/anti-ecológicos (mata bichos) & & & 1 & 9,1 & 1 & 4,2 \\
\hline subtotal & 1 & 7,7 & 2 & 18,2 & 3 & 12,5 \\
\hline \multicolumn{7}{|l|}{ Não definido } \\
\hline dá respostas vagas & 1 & 7,7 & 2 & 18,2 & 3 & 12,5 \\
\hline diz não saber & & & 1 & 9,1 & 1 & 4,2 \\
\hline subtotal & 1 & 7,7 & 3 & 27,3 & 4 & 16,7 \\
\hline Subtotal família má & 13 & 100 & 11 & 100 & 24 & 100 \\
\hline
\end{tabular}

Quando comparadas por idade, verificou-se a predominância da função de socializadora em $37,5 \%$ das verbalizações de crianças de cinco anos; $30,8 \%$ das de quatro anos e, também, pela única criança de três anos respondente. As crianças das três idades destacaram, ainda, o cumprimento da função de cuidadora. Apenas crianças de quatro anos atribuíram à questão respostas vagas, não definindo o que a família boa faz.

Em relação à questão sobre o que faz uma família má, a única criança de três anos que a respondeu identificou a própria família como não sendo má; aos quatro anos, prevaleceu o não cumprimento adequado da função socializadora, porque a família pune $(45,5 \%)$; crianças de cinco anos também destacaram o não cumprimento adequado dessa função $(58,4 \%)$, porém sob aspectos mais diversificados.

O percentual de verbalizações das crianças mostrouse maior, em geral, quando responderam a "o que faz uma família" (53,8\%) do que a "o que é família" (46,2\%). Apenas $27,3 \%$ das crianças não responderam à questão "o que é uma família" e 30,3\% a "o que uma família faz". No entanto, a pergunta "o que é uma família má" foi a que teve maior freqüência de não respondentes $(84,8 \%)$, seguida de "o que é uma família boa" (72,7\%). Muitas crianças também não responderam sobre o que faz uma família boa $(60,6 \%)$ e má $(54,5 \%)$.

Em relação à idade, foram as crianças de três anos que não responderam às questões, em maior proporção. Entre as respondentes desta faixa etária, as do Grupo B verbalizaram mais do que as do Grupo A; entre as de quatro e cinco anos ocorreu o oposto, com as crianças do Grupo A verbalizando aproximadamente o dobro das crianças do Grupo B. A média de verbalizações, por idade e grupo, encontra-se ilustrada na Figura 1. 


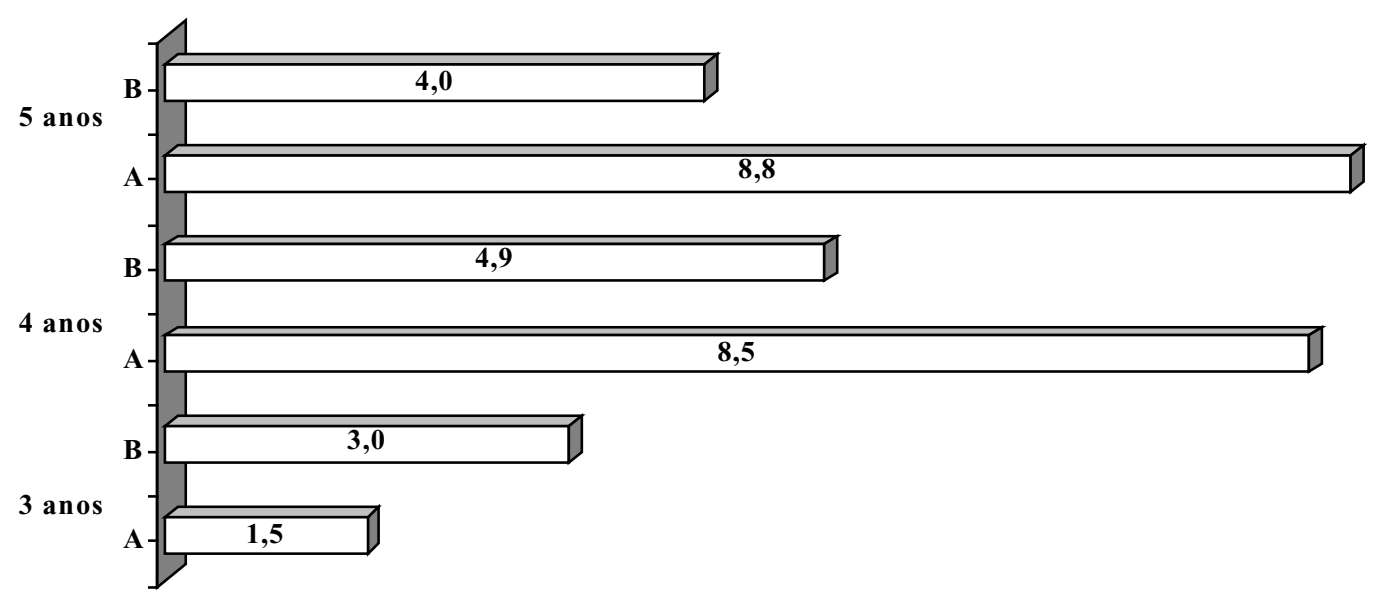

Médias das verbalizações

Figura 1. Média de verbalizações das crianças sobre família, por grupo e idade.

\section{Apresentação de aspectos característicos e definidores de família e opiniões das crianças}

Frente aos aspectos característicos e definidores de família, apresentados às crianças na segunda parte da entrevista, conforme descrito na seção do método, as crianças eram questionadas sobre a possibilidade de que as pessoas integrantes da história relatada formassem ou não uma família.

Na primeira questão, as crianças de três anos dividiramse entre concordar e discordar; $70 \%$ das crianças de quatro anos e $85,7 \%$ das crianças de cinco anos concordaram que o grupo apresentado pudesse ser uma família. Na segunda questão, as crianças de três anos concordaram que o grupo pudesse formar uma família; mais crianças de quatro $(70 \%)$ e de cinco anos $(71,4 \%)$ concordaram do que discordaram, embora $28,6 \%$ das crianças de cinco anos não tenham respondido à questão.

Em síntese, para muitas crianças deste estudo, um grupo de pessoas que não estivesse totalmente envolvido ou passasse pouco tempo junto, desde que as pessoas fizessem algumas coisas juntas e umas para as outras, poderiam formar uma família. A aceitação do grupo como família aumentou em proporção à idade.

\section{Discussão}

Mudanças sociais e econômicas são indicadas, na literatura, como propulsoras da ocorrência de ajustes nos papéis desempenhados na família e em sua conceituação (Cerqueira-Silva e cols., 2008). Esse fato é identificado nas concepções de família das crianças pré-escolares participantes deste estudo, especialmente ao utilizarem o critério da composição familiar em suas definições.

Ao citarem a composição familiar, as crianças referiramse, principalmente, às relações biológicas entre seus membros, mas raramente descrevendo a família como formada apenas pelo núcleo familiar, como relatado em estudos mais remotos (Moore e cols., 1977; Powell e cols., 1981). Elas incluíram, em suas definições, membros da família extensa, pessoas com relações não-biológicas e animais de estimação, indicando um reconhecimento semelhante ao descrito na literatura, a respeito da variabilidade das famílias contemporâneas e, em específico, das famílias brasileiras (Stratton, 2003; Torres \& Dessen, 2007). Tais dados confirmam a constatação teórica de que a família não mais é vista apenas como um sistema nuclear, tendo incorporado aqueles que fazem parte de sua rede de relações e com quem têm afinidades (Petzold, 1996). Eles também corroboram os resultados de estudos empíricos mais recentes, tanto em diferentes culturas (Georgas e cols., 2007) quanto com crianças (Morrow, 1998; Roe e cols., 2006).

Diferenças e similaridades nas concepções das crianças decorrentes de seu desenvolvimento foram claramente verificadas nas respostas das crianças deste estudo. Algumas crianças, especialmente aos três anos, apresentaram baixa freqüência de verbalizações a respeito da família - um conceito relativamente abstrato - e dificuldades para atribuir definições aos pólos bom/mau, conforme descrito por Morrow (1998). As crianças pouco maiores (de quatro e, principalmente, cinco anos), por sua vez, já utilizaram algumas definições abstratas, indicando a sua maior flexibilidade para o desenvolvimento do conceito de família.

As concepções das crianças também refletem as próprias mudanças pelas quais passam a sociedade e a família contemporâneas. A questão das mudanças remete aos modos de vida das famílias, incluindo o envolvimento de pais e mães na divisão de tarefas domésticas e de cuidados com os filhos. Neste sentido, as crianças apontaram as mães como aquelas que fazem muitas coisas, principalmente aquelas que não tem ocupação remunerada (Grupo B). No entanto, demonstraram reconhecer, também, a proximidade e o envolvimento do pai nos cuidados a elas dispensados e nos afazeres domésticos, em ambos os grupos, mas especialmente entre crianças cujas mães possuíam ocupação remunerada (Grupo A). Ou seja, as crianças do Grupo A, cujas mães possuíam ocupação remunerada, 
percebiam mais a existência de divisão de tarefas entre as mães e os pais do que as crianças do Grupo B.

Mudanças semelhantes vêm sendo apontadas na literatura. Com o aumento do trabalho remunerado das mães, um número crescente de pais compartilha com elas, ou assume para si, a responsabilidade de educar os filhos, buscando se adequar à realidade atual. Isso tende a conduzir o pai a uma maior proximidade e participação na educação do filho, embora uma maior responsabilidade ainda seja, muitas vezes, atribuída à mãe (Dessen \& Torres, 2002; Wagner, Predebon, Mosmann, \& Verza, 2005).

Ainda em relação ao envolvimento de ambos os genitores com ocupações remuneradas, outra possibilidade de cuidados dispensados à criança caracteriza-se pela busca de ajuda de outras pessoas, como parentes, empregadas e instituições de educação (Simionato-Tozo \& Biasoli-Alves, 1998). É importante destacar que ambas as situações foram verificadas, especialmente, nas famílias do Grupo A deste estudo.

Logo, a maior inserção da mulher no mercado de trabalho tem gerado mudanças, também, na percepção que a criança tem das funções desempenhadas na família. Isto se dá, especificamente, quanto à opinião das crianças sobre os papéis exercidos pelo pai e pela mãe, indicando um ajuste positivo delas às atuais condições, enquanto os adultos têm sido apontados como relutantes em ultrapassar papéis familiares tradicionais (Gomes \& Resende, 2004).

À sua maneira, as crianças participantes descrevem o que ocorre internamente nas famílias, tendo se mostrado importante, para elas, o envolvimento do adulto (especialmente mãe e pai) em seus cuidados; por outro lado, as suas verbalizações indicam que não aprovam práticas punitivas como parte de uma família considerada boa. Neste contexto, é também importante conhecer a percepção dos estilos e das práticas parentais das crianças (Weber, Prado, Viezzer, \& Brandenburg, 2004). A ampliação deste conhecimento poderia auxiliar na prevenção de problemas advindos de tais práticas que, de acordo com Dessen e Szelbracikowski (2006), fazem parte da etiologia da manifestação de comportamentos agressivos e de oposição pela criança, que, por sua vez, podem interferir no seu processo de aprendizagem.

Se consideradas do ponto de vista da perspectiva do desenvolvimento familiar, as famílias participantes passavam por mudanças necessárias ao seu estágio de desenvolvimento, próprias de famílias com filhos pequenos, como: a) ajustes no sistema conjugal para criar espaço para o(s) filho(s), b) concentração de esforços nas tarefas de educação, financeiras e domésticas; e, ainda, c) um realinhamento de papéis e relacionamentos com as famílias ampliadas (Carter \& McGoldrick, 1995). Estas mudanças se refletem nas concepções das crianças, que foram consistentes com a definição ecopsicológica de família (Petzold, 1996).
Portanto, a família é um conceito vivenciado e compartilhado pelas crianças, ao interagirem, durante o seu processo de socialização. Embora as crianças e suas famílias pareçam estar, paulatinamente, se ajustando às atuais condições da vida moderna, ainda há necessidade do desenvolvimento de políticas públicas em seu favor (Stratton, 2003). Além disso, faz-se necessário expandir a compreensão teórica e empírica sobre as atuais concepções de família, uma vez que esta desempenha um papel preponderante no desenvolvimento humano.

Assim, os resultados apresentados neste estudo possibiltam compreender melhor as concepções que as crianças têm de família, uma vez que há poucos estudos empíricos que considerem a sua opinião, conforme relatado na seção introdutória deste artigo. O reconhecimento da participação ativa da criança é recente e a relevância de suas formas de comunicação e de suas ações vêm sendo apontadas (Kreppner, 2003; Percy, 2003). Além disso, as concepções de família ora apresentadas pelas crianças podem ser utilizadas como base de comparação em situações de intervenção direcionadas às crianças pequenas e suas famílias.

Apesar da relevância dos dados, é preciso ter cautela, na medida em que, nas entrevistas, apesar da utilização de artifícios lúdicos para atrair a atençao das crianças, algumas delas se distraíram com a presença do cenário, especialmente as mais novas. Isto pode ter acarretado na não manutenção de um nível adequado de atenção, prejudicando as respostas das crianças mais novas, o que também foi destacado por Souza (2006), em situação semelhante com crianças pequenas. Na literatura, o uso de figuras representando os conceitos explorados, encenações de histórias com bonecos ou desenhos feitos pelas crianças são citados como possibilidades de complementação à entrevista, tendo em vista captar mais amplamente as concepções de crianças pequenas (Roe e cols., 2006). Também é importante destacar a necessidade de que o entrevistador e o pesquisador estejam bem preparados para lidar com a criança, e para interpretar suas verbalizações (Montandon, 2005).

Concluímos, reiterando a importância de que, em pesquisas com e sobre a família, a criança seja escutada, ainda que apresente um conceito pautado na sua própria família, pois com ela convive e nela aprende valores e crenças. A convivência das crianças com a família extensa, com outras pessoas adultas e com outras crianças em seu dia-a-dia possibilita que se tornem fontes importantes de informação, na pesquisa. Uma visão sistêmica e global do funcionamento das famílias só é possível quando se considera as opiniões de todos os subsistemas familiares, incluindo aqueles em que a criança participa ativamente (Dessen \& Lewis, 1998). Além disso, é fundamental que os estudos futuros examinem as concepções de de família das crianças, mas em uma perspectiva longitudinal e transcultural. 


\section{Referências}

Carter, B., \& McGoldrick, M. (1995). As mudanças no ciclo de vida familiar (2a ed). Porto Alegre: Artes Médicas.

Cerqueira-Silva, S., Oliveira, N. R., \& Dessen, M. A. (2008). A dinâmica das famílias brasileiras em foco: Passado e presente (Relatório de Pesquisa). Brasília: Laboratório de Desenvolvimento Familiar, Universidade de Brasília.

Dessen, M. A. (1999). Questionário sociodemográfico da família (Relatório de Pesquisa). Brasília: Laboratório de Desenvolvimento Familiar, Universidade de Brasília.

Dessen, M. A., \& Biasoli-Alves, Z. M. M. (2001). O estudo da família como base para a promoção da tolerância. In Z. M. M. Biasoli-Alves \& R. Fischmann (Orgs.), Crianças e adolescentes: Construindo uma cultura da tolerância (pp. 183-193). São Paulo: EDUSP.

Dessen, M. A., \& Cerqueira-Silva, S. (2009). Desenvolvendo sistemas de categorias com dados de entrevistas. In L. Weber \& M. A. Dessen (Orgs.), Pesquisando a familia: Instrumentos para coleta e análise de dados (pp. 43-56). Curitiba, PR: Juruá.

Dessen, M. A., \& Lewis, C. (1998). Como estudar a família e o pai? Paidéia (Ribeirão Preto), 8, 105-121.

Dessen, M. A., \& Szelbracikowski,A. C.(2006). Estabilidades e mudanças em padrões familiares de crianças com problemas de comportamento exteriorizado. Paidéia (Ribeirão Preto), 16, 71-80.

Dessen, M. A., \& Torres, C. (2002). Family and socialization factors in Brazil: An overview. In W. J. Lonner, D. L. Dinnel, S. A. Hayes, \& D. N. Sattler (Orgs.), OnLine readings in psychology and culture. Bellingham, USA. Recuperado em 04 agosto 2008, de http://www.wwu. edu/ culture

Georgas, J., Berry, J. W., \& Kağitçibaşi, Ç. (2007). Synthesis: How similar and how different are families across cultures? In J. Georgas, J. W. Berry, F. J. R. van de Vijver, Ç. Kağitçibaşi, \& Y. H. Poortinga (Orgs.), Families across cultures: A 30-nation psychological study (pp. 186-240). New York: Cambridge University Press.

Gomes, A. J. S., \& Resende, V. R. (2004). O pai presente: o desvelar da paternidade em uma família contemporânea. Psicologia: Teoria e Pesquisa, 20, 119-125.

Hodkin, B., Vacheresse, A., \& Buffett, S. (1996). Concepts of family: Methodological issues in assessing perceived family memberships. In M. Cusinato (Orgs.), Research on family resources and needs across the world (pp. 4554). Milano, Italia: LEDEdizioni Universitarie.

Kreppner, K. (2003). Social relations and affective development in the first two years in family contexts. In J. Valsiner \& K. J. Connolly (Orgs.), Handbook of Developmental Psychology (pp. 194-214). Londres: Sage.
Laible, D., Carlo, G., Torquati, J., \& Ontai, L. (2004). Children's perceptions of family relationships as assessed in a Doll Story Completion Task: Links to parenting, social competence, and externalizing behavior. Social Development, 13, 551-569.

Montandon, C. (2005). As práticas educativas parentais e a experiência das crianças. Educação e Sociedade, 26, 485-507. Recuperado em 01 fevereiro 2007, de http:// www.scielo.br/scielo.php

Moore, N. V., Bickhard, M. H., \& Cooper Jr., R. G., (1977). The child's development of the concept of family [Resumo]. Biennial Meeting of the Society for Research in child Development. New Orleans, LA. Recuperado em 23 janeiro 2008, de http://www.eric.ed.gov

Morrow, V. (1998). Understanding families: Children's perspectives [Resumo]. London: National Children's. Recuperado em 23 janeiro 2008, de http://www.jrf.org. uk

Percy, M. S. (2003). Feeling loved, having friends to count on, and taking care of myself: Minority children living in poverty describe what is 'special' to them. Journal of Children and Poverty, 9, 55-70.

Petzold, M. (1996). The psychological definition of "the family". In M. Cusinato (Org.), Research on family: Resources and needs across the world (pp. 25-44). Milão: LED-Edicioni Universitarie.

Powell, J., Wiltcher, B. J., Wedemeyer, N. V., \& Claypool, P. L. (1981). The young child's developing concept of family. Family and Consumer Sciences Research Journal, 10, 137-149. Recuperado em 15 novembro 2007, de http://fcs.sagepub.com

Roe, A., Bridges, L., Dunn, J., \& O'Connor, T. G. (2006). Young children's representations of their families: A longitudinal follow-up study of family drawings by children living in different family settings. International Journal of Behavioral Development, 30, 529- 536. Recuperado em 08 abril 2008, de http://jbd.sagepub.com

Shermerhorn, A. C., Cummings, E. M., \& Davies, P. T. (2008). Children's representations of multiple family relationships: Organizational structure and development in early childhood. Journal of Family Psychology, 22, 89-101.

Simionato-Tozo, S. M. P., \& Biasoli-Alves, Z. M. M. (1998). O cotidiano e as relações familiares em duas gerações. Paidéia (Ribeirão Preto), 8, 137-150.

Stratton, P. (2003). Contemporary families as contexts for development. In J. Valsiner \& K. Connolly (Orgs.), Handbook of Developmental Psychology (pp. 333-357). Londres: Sage.

Souza, D. H. (2006). Falando sobre a mente: Algumas considerações sobre a relação entre linguagem e teoria da mente. Psicologia: Reflexão e Crítica, 19, 387-394. 
Torres, C., \& Dessen, M. A. (2007). The Brazilian jeitinho: Brazil's sub-cultures, its diversity of social contexts, and its family structures. In J. Georgas, J. W. Berry, F. J. R. van de Vijver, Ç. Kağitçibaşi, \& Y. H. Poortinga (Orgs.), Families across cultures: A 30-nation psychological study (pp. 259-266). New York: Cambridge University Press.

Tudge, J. (2001). Estudando a criança e a família em seu contexto: Para uma abordagem cultural da tolerância. In Z. M. M. Biasoli-Alves \& R. Fischmann (Orgs.), Crianças e adolescentes: Construindo uma cultura da tolerância (pp. 65-77). São Paulo: EDUSP.

Valsiner, J. (2000). Structure and dynamics of family/kinship groups, and marriage forms. In J. Valsiner (Org.), Culture and human development: An introduction (pp. 86-117). London/California/New Delhi: Sage.

Wagner, A., Predebon, J., Mosmann, C., \& Verza, F. (2005). Compartilhar tarefas? Papéis e funções de pai e mãe na família contemporânea. Psicologia: Teoria e Pesquisa, 21, 181-186.

Weber, L. N. D., Prado, P. M., Viezzer, A. P., \& Brandenburg, O. J. (2004). Identificação de estilos parentais: O ponto de vista dos pais e dos filhos. Psicologia: Reflexão $e$ Crítica, 17, 323-332.

Maria Auxiliadora Dessen é Professora Associada II da Universidade de Brasília (UnB), Coordenadora do Laboratório de Desenvolvimento Familiar e Professora no Programa de Pós-graduação em Processos de Desenvolvimento Humano e Saúde do Instituto de Psicologia da UnB.

Patrícia Cristina Campos Ramo é Mestre em Psicologia pelo Programa de Pós-graduação em Processos de Desenvolvimento Humano e Saúde do Instituto de Psicologia da Universidade de Brasília.

Recebido: 14/01/2009

$1^{a}$ revisão: $11 / 08 / 2009$

$2^{a}$ revisão: 09/10/2009

$3^{a}$ revisão: $21 / 01 / 2010$

Aceite final: 09/02/2010 